\title{
Surface Modification of the UHMW-PMMA Film Processing by Solvent Molding
}

\author{
Zhongyan $\mathrm{Du}^{\mathrm{a}}$, Jihu Wang ${ }^{\mathrm{b}}$, Shaoguo Wen ${ }^{\mathrm{c}, *}$, Pengzhu Wang ${ }^{\mathrm{d}}$, Dongdong Zhang ${ }^{\mathrm{e}}$, Changle Yin $^{\mathrm{f}}$ \\ College of Chemistry and Chemical Engineering, hanghai University of Engineering Science, Shanghai 201620, P. R. China. \\ a zhongyan_du@163.com, ${ }^{b}$ wangjihu@163.com, ${ }^{\mathrm{c},}$ sg_wen@qq.com, ${ }^{\mathrm{d}} 1036647459 @ q q . c o m,{ }^{\mathrm{e}} 406187213 @ q q . c o m$, \\ 565325414@qq.com.
}

\begin{abstract}
This paper have compared the properties of UHMW-PMMA film with GMW-PMMA film in terms of contact angle, gas permeability, heat resistance, surface resistance changes by surface modification of UHMW-PMMA film like thermal treatment, acidation and alkalization modification. All the datas showed that UHMW-PMMA film has a better performance than GMW-PMMA using in the dental material.
\end{abstract}

Keywords-film; contact angle; gas permeability; heat resistance; surface modification.

\section{INTRODUCTION}

Poly (methyl methacrylate) (PMMA) has good biocompatibility, high reliability, and relative ease of manipulation, low toxicity, and higher transparency than glass with regard to light. PMMA has been used widely as a dental material for the fabrication of complete denture bases ${ }^{[1]}$. While the molecular weight of poly methyl methacrylate being low, the poly methyl methacrylate shows poor chemical resistance and short high temperature ${ }^{[2]}$. With the developing of polymerization initiated by the plasma technology, we can get so-called Ultra high molecular weight PMMA which is a linear and solvent soluble polymer $^{[3-4]}$. The molecular weight of the generated PMMA increases with the time of polymerization increase. The advantage of ultra high molecular weight PMMA not only maintains the mechanical strength of long time at a high temperature but also the deformation is little. In addition, it still has strong abrasion resistance and anti-scratch hardness ${ }^{[5]}$. The completely free polymerization initiator, stabilizer or other low molecular compounds, and is transparent good long-term stability, so that the ultra high molecular weight PMMA is safe which can be applied to the field of artificial teeth, artificial bone and other biomedical polymer materials or materials used for communication ${ }^{[6]}$. The performance of UHMW-PMMA film prepared by solvent dissolved was characterized and the methods of surface modification were investigated in this article.

\section{EXPERIMENTAL}

\section{A. Reagent}

PMMA having an average molecular weight of $\sim 996,000$ as measured by gel permeation chromatography was purchased from Guangdong Province Dongguan City Benz Plastic Co. Ltd. Analytical reagent grade Tetrahydrofuran,
98\% concentrated $\mathrm{H}_{2} \mathrm{SO}_{4}, \mathrm{HCl}$, and $\mathrm{KOH}$ were purchased from Sinopharm Chemical Reagent Co., Ltd; Sulfonated grapheme, Suzhou Gaotong New Materials Co., Ltd.

\section{B. Preparation Of UHMW-PMMA Film}

At room temperature, weighed a certain amount of UHMW-PMMA powder and placed in a glass dish, then poured the solvent of Tetrahydrofuran for dissolution. Due to the molecular weight is too high; the powder dissolved slowly .we can put the dish in a shaker until the powder completely dissolved. After the solvent has evaporated, the film formed (determine through quality comparison before and after the film formation), began testing and characterization. Using the same method dissolve the powder of general molecular weight PMMA.

\section{Surface Modification Of UHMW-PMMA Film}

- Acidation and alkalization modification. The films were soaked in designed concentration of acid and alkaline for $24 \mathrm{~h}$ with temperature maintaining at $25^{\circ} \mathrm{C}$. And then the samples were washed at least three times using water and dry in oven at $45{ }^{\circ} \mathrm{C}$. The changes of mass and surface resistance for the film were investigated.

- Sulfonated graphene modified. At room temperature, through the solvent Tetrahydrofuran as a solvent UHMWPMMA and Sulfonated graphene powder body, the glass is placed on the culture dish forming film and films before and after weight by weighing to determine the solvent volatilizes completely.

\section{CHARACTERIZATION}

Thickness tester (CHY-CA) was made by Lab think Instruments CO., LTD; The constant temperature brteding shaker(Shanghai Baixin Instruments CO., LTD,TS-200B) used at $25^{\circ} \mathrm{C}$; Gas permeability was measured by Gas permeability Tester (Lab think Instruments CO., LTD, BTY$\mathrm{B} 1 \mathrm{P}$ ) according to GB/T 1038-2000; The contact angle was measured by Contact angle measuring instrument (Shanghai Zhongchen Digital Technology Apparatus CO., LTD, JC2000D2A); Thermogravimetic analysis (TGA) (LINSEIS Instruments International, STA PT-1000) was carried out at the rate of temperature $10{ }^{\circ} \mathrm{C} / \mathrm{min}$ under Nitrogen protection; Surface resistance was measured by high insulation resistance measurement instrument (Shanghai ANBIAO Electronic CO., LTD.) according to GB/T 14101989. 


\section{RESULTS AND DISCUSSION}

\section{A. Contact Angle}

The contact angle is used to indicate the degree of wetting of a solid; Refers to the tangent at the gas, liquid and solid three-phase intersection place for gas - liquid interface through the liquid and solid - liquid over the boundary between the angle $\theta$ which is a measure of the degree of wetting. If the angle $\theta<90^{\circ}$, the solid surface is hydrophilic, and the smaller of the angle, the better the wettability is expressed; if the angle $\theta>90^{\circ}$, the solid surface is hydrophobic, and not readily wetted liquid solid, but easy to move on the surface ${ }^{[7]}$. Fig 1 is the contact angle of UHMWPMMA film and GMW-PMMA film, and Table 1 is the surface tension of the two kind films.

The contact angle of the water is an important index of UHMW-PMMA properties, UHMW-PMMA as the dental material. The greater the contact angle, the better the hydrophobicity, the more time of the dental powder material.

Fig. 1 and table 1 show the results of water contact angle measurements of the UHMW-PMMA sample and GMWPMMA, the contact angle of UHMW-PMMA is nearly $90^{\circ}$, and the hydrophobicity and the surface tension of the two surfaces are higher than GMW-PMMA. Because the material is often in the mouth soaking in water, need a good hydrophobic property. All the records show that UHMWPMMA is more advantageous than GMW-PMMA in dental materials and longer service life.

\section{B. Gas Permeability}

Gas permeability is an important physical property of the performance of the film, fabric, coating and other polymer materials. In a certain pressure difference condition, the unit time is expressed by the gas quantity of the sample unit square. And it is related to the structure, phase state and molecular motion of the polymer. We know the data of permeability and permeability coefficient are smaller, the better barrier property ${ }^{[8]}$. The Sulfonated-graphene possesses a rare layer or even monolayer has good barrier property. When the solution is formulating, the trace amount of Sulfonated-graphene is added. Table 2 is some gas permeability dates of three kind samples.

From table 2 we can find GMW-PMMA film has high permeability and permeability coefficient. The permeability and permeability coefficient of UHMW-PMMA film respectively is $387.139 \mathrm{~cm}^{3} / \mathrm{m}^{2} \cdot 24 \mathrm{~h} \bullet 0.1 \mathrm{MPa}, \quad 6.616 \times 10^{-11}$ $\mathrm{cm}^{3} \cdot \mathrm{cm} / \mathrm{cm}^{2} \cdot \mathrm{s} \cdot \mathrm{cm} \cdot \mathrm{Hg}$; they are better than data of GMWPMMA film. Permeability become 127.85 $\mathrm{cm}^{3} / \mathrm{m}^{2} \cdot 24 \mathrm{~h} \cdot 0.1 \mathrm{MPa}$ and permeability coefficient is $3.052 \times 10^{-11} \mathrm{~cm}^{3} \cdot \mathrm{cm} / \mathrm{cm}^{2} \cdot \mathrm{s} \bullet \mathrm{cm} \cdot \mathrm{Hg}$. Gas permeability has changed a lot and become too better than other two kinds (UHMW-PMMA and GMW-PMMA); this result is owing to Sulfonated-graphene possessing a rare layer or even monolayer structure. Therefore, we may be able to add trace amount of Sulfonated-graphene into UHMW-PMMA as a dental material to improve the life length.

\begin{tabular}{|c|c|c|}
\hline Sample & $\begin{array}{c}\text { contact angle } \\
\left({ }^{\circ}\right)\end{array}$ & $\begin{array}{c}\text { solid-liquid interfacial tension } \\
(\mathbf{m N} / \mathbf{m})\end{array}$ \\
\hline UHMW-PMMA & 88.60 & 37.46 \\
\hline GMW-PMMA & 68.00 & 12.93 \\
\hline
\end{tabular}

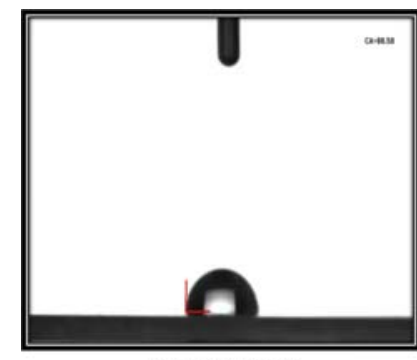

(a) HUMW-PMMA

Figure 1. The contact angle of the films

TABLE II. THE DATA OF GAS PERMEABILITY

\begin{tabular}{|c|c|c|}
\hline Sample & $\begin{array}{c}\text { permeability } \\
(\mathrm{cm} 3 / \mathrm{m} 2 \cdot 24 \mathrm{~h} \cdot 0.1 \mathrm{M} \\
\mathrm{Pa}\end{array}$ & $\begin{array}{c}\text { permeability coefficient } \\
(\mathrm{cm} 3 \cdot \mathrm{cm} / \mathrm{cm} 2 \cdot \mathrm{s} \cdot \mathrm{cm} \cdot \mathrm{Hg}\end{array}$ \\
\hline UHMW-PMMA & 387.139 & $6.616 \times 10^{-11}$ \\
\hline GMW-PMMA & 404.502 & $6.922 \times 10^{-11}$ \\
\hline UHMW-PMMA+SG & 127.850 & $3.052 \times 10^{-11}$ \\
\hline
\end{tabular}

\section{Heat Resistance}

In the usage of wear resistant occasion, UHMW-PMMA will elevate temperature because friction. Heat build-up causes the strength degradation as a result of thermal degradation or chain breaking ${ }^{[9]}$. So it is very important to determine the heat decomposition temperature. TGA curves of UHMW-PMMA film is shown in Fig. 2.

Fig.2 shows the experimental TG curves of sample a (UHMW-PMMA) and sample b (GMW-PMMA). The sample a of UHMW-PMMA weight loss occurs between $295-467^{\circ} \mathrm{C}$. When the temperature rose to $470^{\circ} \mathrm{C}$, the residual is $0 \%$. It is showed the better plasticizing in the process of prepared film at the lower initial temperature of dissociation. For example, the UHMW-PMMA film degrade at $295^{\circ} \mathrm{C}$ while the GMW-PMMA film at $275^{\circ} \mathrm{C}$. Reduction in molecular weight leads to lower initial temperature of dissociation; final temperature of dissociation in the two areas are same which means degradation in the process has little influence to thermal stability of UHMW-PMMA film. Minimum decomposition temperature is $295^{\circ} \mathrm{C}$ which shows pyrolysis temperature of film is relatively high. This temperature has great effect on the resistance to thermal degradation caused by overheating because of instantaneous friction heat production. 


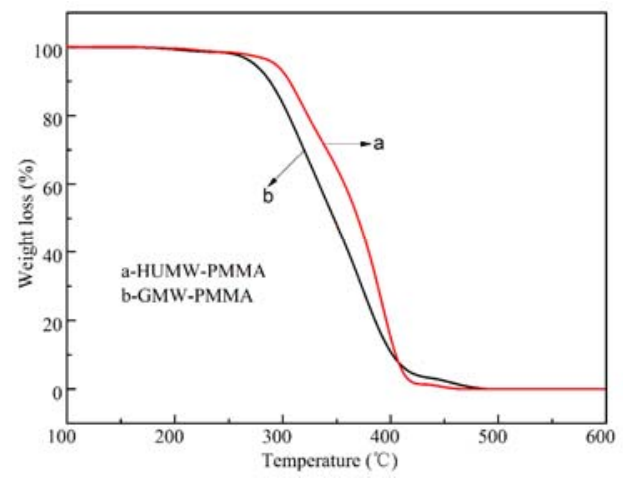

Figure 2. TGA curves of UHMW-PMMA film

\section{Thermal Treatment}

Synthetic polymer materials have more superior performances like electrical insulation than natural polymer materials. We have raised the temperature while testing the change of surface resistance that determines the heat synthetic polymer materials have more superior performances like electrical insulation than natural polymer materials. We have raised the temperature while testing the change of surface resistance that determines the heat resistance of HUMW-PMMA as dental materials in the mouth. Table 3 shows the Change $R_{\mathrm{s}}$ of UHMW-PMMA film and GMW-PMMA film at the temperature from 25 to $65^{\circ} \mathrm{C}$ at intervals of $10^{\circ} \mathrm{C}$ for $1 \mathrm{~h}$ at every temperature point.

TABLE III. CHANGE IN SURFACE RESISTANCE BY THERMAL TREATMENT OF FILMS

\begin{tabular}{|c|c|c|c|c|c|}
\hline $\operatorname{Rs}(\Omega)$ of Items & 25 & 35 & 45 & 55 & 65 \\
\hline $\begin{array}{l}\text { UHMW- } \\
\text { PMMA }\end{array}$ & $9.0 \times 10^{13}$ & $6.3 \times 10^{13}$ & $3.8 \times 10^{13}$ & $5.0 \times 10^{13}$ & $2.5 \times 10^{13}$ \\
\hline GMW-PMMA & $2.3 \times 10^{14}$ & $8 \times 10^{13}$ & $5.0 \times 10^{13}$ & $3.8 \times 10^{13}$ & $1.3 \times 10^{13}$ \\
\hline
\end{tabular}

According to table 3 , as one kind of polymer materials has high volume resistance, and low conductivity. Not only UHMW-PMMA film but also GMW-PMMA film the Rs of change are both little; this illustrates even at the mouth temperature, and the material will hardly change.

\section{E. Acidation And Alkalization Modification}

We all know that our saliva often appear faintly acid in our oral cavity, also drinking alkaline water. Therefore, the materials used for dental surface should resistant acid base and alkaline base. The antistatic property of the surface is expected to be a little or even no change by acidation and alkalization modification (impregnation method) through increasing surface groups of UHMW-PMMA film. The surface resistance of the films is in Table 4.

Table 4 shows that the changes in surface resistance both of UHMW-PMMA film and GMW-PMMA film soaked in acid or alkaline solution at room temperature for $24 \mathrm{~h}$ then dry at $45^{\circ} \mathrm{C}$. Film surface resistance has a little upward trend after modified by low concentration of acid and alkaline solution. These datas are useful for UHMW-PMMA film used as a dental material. The data indicate that will not affect the mechanical properties of the films while appear in our oral cavity .

TABLE IV. CHANGE IN SURFACE RESISTANCE BY ACIDATION AND ALKALIZATION MODIFICATION

\begin{tabular}{|l|l|l|l|l|}
\hline \multicolumn{2}{|c|}{ Items } & $\begin{array}{c}\mathbf{H}_{2} \mathbf{S O}_{4} \\
(\mathbf{1 0 w t} \%)\end{array}$ & $\begin{array}{c}\text { HCl } \\
(\mathbf{1 0 w t} \%)\end{array}$ & $\begin{array}{c}\text { KOH } \\
(\mathbf{1 0 w t} \%)\end{array}$ \\
\hline $\begin{array}{l}\text { Before } \\
\text { modified } \\
R_{\mathrm{S}}(\Omega)\end{array}$ & UHMW-PMMA & $7.5 \times 10^{12}$ & $4.0 \times 10^{12}$ & $1.3 \times 10^{13}$ \\
\cline { 2 - 5 } $\begin{array}{l}\text { After } \\
\text { modified } \\
R_{\mathrm{S}}(\Omega)\end{array}$ & GMW-PMMA & $9.0 \times 10^{12}$ & $3.5 \times 10^{12}$ & $1.8 \times 10^{13}$ \\
\cline { 2 - 5 } & GHMW-PMMA & $8.5 \times 10^{12}$ & $5.0 \times 10^{12}$ & $2.0 \times 10^{13}$ \\
\hline
\end{tabular}

\section{CONCLUSIONS}

Through the characterization and modification of the film, it can be found:

- In terms of contact angle, gas permeability, heat resistance of UHMW-PMMA film and GMW-PMMA film, the former film has a better performance than the latter one using in the dental material.

- After added Sulfonated-graphene into UHMWPMMA, permeability become $127.850 \mathrm{~cm}^{3} / \mathrm{m}^{2} \cdot 24 \mathrm{~h} \cdot 0.1 \mathrm{MPa}$ and permeability coefficient permeability coefficient is $3.052 \times 10^{-11} \mathrm{~cm}^{3} \cdot \mathrm{cm} / \mathrm{cm}^{2} \cdot \mathrm{s} \bullet \mathrm{cm} \cdot \mathrm{Hg}$. Gas permeability has changed a lot and become too better than pure UHMWPMMA film. And permeability coefficient permeability coefficient is $3.052 \times 10^{-11} \mathrm{~cm}^{3} \cdot \mathrm{cm} / \mathrm{cm}^{2} \cdot \mathrm{s} \bullet \mathrm{cm} \cdot \mathrm{Hg}$. Gas permeability has changed a lot and become too better than pure UHMW-PMMA film.

- Modified the surface of UHMW-PMMA film by the method like thermal treatment, acidation and alkalization modification, and the surface resistance changed very little. This indicates that UHMW-PMMA film as a dental material is suited to use in our oral cavity.

\section{ACKNOWLEDGMENT}

This work is supported by the Suzhou Gaotong New Materials Co., Ltd., and we are very grateful to you for your valuable support. Also special thanks to Professor Wen and Dr Wang for supporting to this study.

\section{REFERENCES}

[1] H. W. Park, I. Lee. Large pulsed electron beam surface treatment of translucent PMMA. Applied Surface Science, vol. 308, pp. 311-315, 2014.

[2] H. J. Jeon, J.H. Youk, S. H. Ahn, J.H.Choi, K. S. Cho. Synthesis of High Molecular Weight 3-Arm Star PMMA by ARGET ATRP. Macromolecular Research, Vol. 17, No. 4, pp 240-244, 2009.

[3] D. M. Kim, J. Kim, S.S. Park. Surface modification of the patterned Al6061/SUS304 metal plates using the large electron beam.Appl. Surf. Sci, vol. 261, pp. 458, 2012.

[4] J. Faltermeier, P. Simon, C. Reicheneder. The influence of electron beam irradiation on color stability and hardness of aesthetic brackets. Eur. J. Orthod, vol. 34, No. 4, pp. 427-431, 2012. 
[5] T. Tippo, C. Thanachayanont, P. Muthitamongkol. The effects of solvents on the properties of ultra-thin poly (methyl methacrylate) films prepared by spin coating. Thin Solid Films, vol. 546, pp. 180 184, 2013.

[6] T. Jin, Z.W. Zhou, Z.H. Wang. Quasi-static failure behavior of PMMA under combined shear-compression loading. Polymer Testing, vol. 42, pp. 181-184, 2015.

[7] G.M. Shanthini, C. A. Martin, N. Sakthivel, S. C. Veerla, K. Elayaraja, B. S. Lakshmi, et al. Physical and biological properties of the ion beam irradiated PMMA-based composite films. Applied Surface Science, vol. 329, pp. 116-126, 2015.
[8] E. H. Lock, D. M. Delongchamp, S. W. Schmucker, B. Simpkins ,M. Laskoski, S. P. Mulvaney, et al. Dry graphene transfer print to polystyrene and ultra-high molecular weight polyethylene-Detailed chemical, structural, morphological and electrical characterization. CARBON, vol. 86, pp. 288-300, 2015

[9] T. Jin, X.Y. Niu, G.S. Xiao, Z. H. Wang, Z. W. Zhou, G.Z, Yuan, et al. Effects of experimental variables on PMMA nano-indentation measurements. Polymer Testing, vol. 41, pp. 1-6, 2015 